\title{
Comparison of the robustness and functionality of three adrenaline auto-injectors
}

This article was published in the following Dove Press journal:

Journal of Asthma and Allergy

17 August 2012

Number of times this article has been viewed

\author{
Andreas Schwirtz \\ Harald Seeger \\ Pharma Consult GmbH, \\ Vienna, Austria
}

Correspondence: Andreas Schwirtz Pharma Consult GmbH, Research and Development, Divischgasse 4, A- 1210 Vienna, Austria

$\mathrm{Tel}+43$ । 29107669

$\mathrm{Fax}+43$ I 291076377

Email a.schwirtz@bmgrp.at
Background: Anaphylaxis is a medical emergency that requires the intramuscular injection of adrenaline using an adrenaline auto-injector (AAI). This study compared the robustness and performance characteristics of three AAIs available in Europe.

Methods: Three AAIs (Jext ${ }^{\circledR}$, EpiPen ${ }^{\circledR}$, and Anapen ${ }^{\circledR}$ ) were tested in terms of the force needed to activate the AAIs, exposed needle length, injection volume, and injection time. Three conditions were used to assess robustness: base conditions, after three successive free-fall drops from $1.5 \mathrm{~m}$, and after a $40 \mathrm{~kg}$ static load challenge. The injection depth and estimated volume of solution delivered into ballistic gelatin were also assessed.

Results: Less force was required to remove the safety cap from Jext and EpiPen than from Anapen under base conditions. The required force was unaffected by free-fall drop tests, whereas the static load test significantly increased the force required to remove the safety cap from Jext (difference from base value $7.7 \mathrm{~N} ; P<0.001$ ) and from EpiPen (difference from base value $30.3 \mathrm{~N} ; P<0.001)$. Two Anapens could not be activated after the free-fall and static load tests. The mean exposed needle length was $15.36 \mathrm{~mm}$ (standard error [SE] 0.04) for Jext, $15.02 \mathrm{~mm}$ (SE 0.05) for EpiPen, and $7.49 \mathrm{~mm}$ (SE 0.15) for Anapen. The mean maximum injection depth in gelatin within 10 seconds was $28.87 \mathrm{~mm}$ (standard deviation [SD] 0.73) for Jext, $29.68 \mathrm{~mm}$ (SD 2.08) for EpiPen, and $18.74 \mathrm{~mm}$ (SD 1.25) for Anapen.

Conclusion: A comparison of the robustness and performance characteristics of the three AAIs showed that cartridge-based devices (Jext and EpiPen) appeared to be significantly more robust and capable of rapidly and consistently delivering the correct dose of adrenaline to the correct tissue compartment than the syringe-based Anapen. Overall, Jext performed better than EpiPen or Anapen following mechanical stress designed to mimic real-world use.

Keywords: anaphylaxis, adrenaline auto-injector, Anapen, EpiPen, Jext, injection depth, reliability

\section{Introduction}

Anaphylaxis is a rapid, severe, and life-threatening systemic allergic reaction. Anaphylaxis occurs as a result of the release of proinflammatory mediators from mast cells and basophils in response to an allergen. The most common allergens that are responsible for inducing anaphylaxis include foods, particularly peanuts, tree nuts, shellfish and fish, cow's milk, eggs, and wheat; medications, most commonly antibiotics; insect stings; and natural rubber latex. ${ }^{1}$

The systemic nature of anaphylaxis means that multiple organs are affected, which include the skin, lungs, gastrointestinal tract, and cardiovascular system, resulting in numerous potentially serious symptoms. ${ }^{2}$ Even those symptoms that are not immediately life-threatening may progress rapidly unless treatment is initiated promptly. However, 
respiratory compromise and cardiovascular collapse are the symptoms that are the most frequent causes of anaphylaxisrelated fatality. ${ }^{3}$

The frequency of anaphylaxis has been estimated to be 50-2000 episodes per 100,000 persons, with a lifetime prevalence of $0.05 \%-2.0 \%$. Attacks of anaphylaxis lead to the death of $0.65 \%-2.0 \%$ of sufferers. It is of concern that the occurrence of anaphylaxis appears to be rising, particularly among younger age groups..$^{3-5}$

Intramuscular administration of adrenaline into the thigh is widely recognized as the first-line medication of choice in anaphylaxis. Administration should take place immediately once a diagnosis of anaphylaxis is suspected. ${ }^{3,6}$ Adrenaline is life-saving in anaphylactic attacks owing to its alpha-1 adrenergic vasoconstrictor effects and its ability to prevent and relieve the airway obstruction caused by mucosal edema, hypotension, and shock. ${ }^{6,7}$ Failure to administer adrenaline promptly is potentially associated with fatality. ${ }^{6}$

Because anaphylaxis most commonly occurs outside a healthcare setting, adrenaline auto-injectors (AAIs) are routinely prescribed to patients previously diagnosed with anaphylaxis or who are at serious risk of anaphylaxis as a result of their allergic history. ${ }^{8}$ The AAI should be carried by the patient at all times to enable rapid self-administration of intramuscular adrenaline at the onset of an anaphylactic attack. Fundamental to the management of the symptoms of anaphylaxis by an AAI is its ability to deliver a full dose of adrenaline to the correct tissue compartment (ie, the thigh muscle) as rapidly as possible, even through clothing. The AAI should also be easy and convenient to use, as well as robust, safe, and reliable enough to withstand real-life storage and use. ${ }^{9}$

The AAIs currently available in Europe $\left(\mathrm{Jext}^{\circledR}, \mathrm{EpiPen}^{\circledR}\right.$, and Anapen ${ }^{\circledR}$ ) are designed to deliver a single dose of $0.15 \mathrm{mg}$ (pediatric) or $0.3 \mathrm{mg}$ (adult) adrenaline in a sterile solution intramuscularly into the vastus lateralis muscle of the thigh. In addition, Anapen is available in a $0.5 \mathrm{mg}$ version (Anapen ${ }^{\circledR}$ 500). EpiPen first became commercially available in Europe in 1994, Anapen in 2003, and Jext in 2010. ${ }^{9,10}$ Unfortunately, differences in the functional and injection properties of the currently available AAIs may lead to variations in their individual abilities to administer adrenaline effectively as and when required. ${ }^{11}$

In this study, the performance of three AAIs designed to deliver $0.3 \mathrm{mg}$ adrenaline was compared: Jext and EpiPen, which are cartridge-based AAIs, and Anapen, a syringebased AAI. The main objectives of the study were to assess the robustness and functionality of the three AAIs, in terms of activation force required, exposed needle length, and injection volume and time. These characteristics were assessed under base conditions, after three successive freefall drops and after a $40 \mathrm{~kg}$ static load challenge, to mimic everyday wear and tear. Further objectives were to estimate the injection depth, the dose delivered, and the distribution of the delivered adrenaline in ballistic gelatin to simulate real-use conditions.

\section{Materials and methods}

The three types of AAI devices used for testing in January 2012 were Jext (Jext $300 \mu \mathrm{g}$ adrenaline solution for injection in prefilled pen; batch 000139318 [expiry date May 2013]; ALK, Hoersholm, Denmark), EpiPen (EpiPen adrenaline autoinjector $0.3 \mathrm{mg}$; batches 0000129760-0000131211 [expiry date September 2011], 0000132207-0000132894 [expiry date January 2012], 0000130394-0000132454 [expiry date October 2011], $0000132207-0000132953$ [expiry date January 2012]; Meda AB, Stockholm, Sweden), and Anapen (Anapen $300 \mu \mathrm{g}$ in $0.3 \mathrm{~mL}$ solution for injection in a prefilled syringe; batches LJR14022012 [expiry date February 14, 2012], TLB10042013 [expiry date April 10, 2013], LFW14022012 [expiry date February 14, 2012], MPD28032012 [expiry date March 28, 2012], and RRT14112012 [expiry date November 14, 2012]; Lincoln Medical Ltd, Salisbury, UK). The three devices are shown in Figure 1.

The methods used for testing the devices were essentially as described in a previous pilot study comparing two AAIs

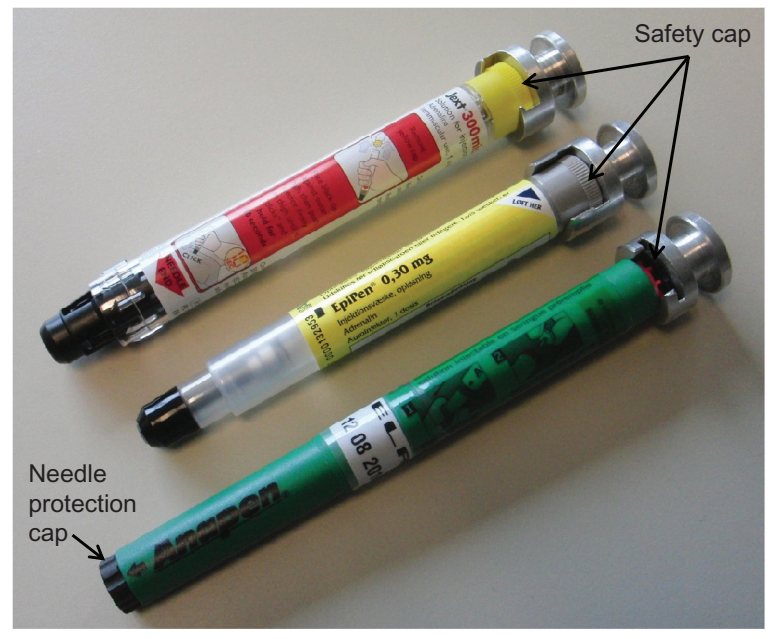

Figure I The three adrenaline auto-injectors compared in the study (Jext, top; EpiPen, middle; Anapen, bottom), with adapters attached for measurement of safety cap removal force.

Note: The safety cap on each adrenaline auto-injector and the needle protection cap on the Anapen must be removed before activation is possible. 
developed for children (Anapen ${ }^{\circledR}$ Junior and EpiPen ${ }^{\circledR}$ Junior). ${ }^{11}$ The different performance tests are briefly described below. All measurements were performed at standard atmospheric conditions (temperature $18^{\circ} \mathrm{C}-28^{\circ} \mathrm{C}$, relative humidity $25 \%-75 \%$ ). All AAIs were maintained under these conditions for at least four hours prior to testing.

\section{Robustness and functionality of AAls}

The first series of tests were related to the robustness and functionality of the AAIs. Measurements of the force required to remove safety mechanisms and to activate the AAIs, and measurements of injection volume, time, and exposed needle length, were carried out under each of three conditions: base conditions (ie, no preconditioning); after free-fall drops, where each device was dropped three times (once horizontally and twice vertically) from a height of $1.5 \mathrm{~m}$ onto a concrete plate, guided by a plastic pipe; and after a static load challenge, where the devices were subject to a 400 Newtons ( $\mathrm{N}$, equivalent to $40 \mathrm{~kg}$ ) static weight for 10 seconds. A $400 \mathrm{~N}$ static weight was selected because this was equivalent to the static load used in the pilot study, ${ }^{11}$ thus enabling comparison of data between the current and the earlier study.

\section{Removal of safety mechanisms}

The force required to remove the safety cap from each AAI was tested using $\mathrm{Zwick}^{\odot}$ single column testing equipment (Zwick Roell, Ulm, Germany), utilizing specially designed adapters to hold the caps (Figure 1).

EpiPen and Jext both have a single safety cap, which must be removed before the device can be activated. Anapen has two caps: a needle cap (which protects the sterile needle of the prefilled syringe) and a safety cap. Because both caps must be removed before Anapen can be used, the force required to remove the needle cap was also measured.

\section{Activation force}

The activation steps described in the summary of product characteristics is slightly different for the individual AAIs (thumb activation for Anapen, swing and jab activation for EpiPen, and place and press activation for Jext). However, in this study each of the AAIs was activated using the same methodology. After each AAI was secured vertically onto the Zwick equipment (via exchangeable adapters that incorporated the different systems), the device was activated by pressing onto its top using a plate surface. The activation force required for each AAI was recorded.

\section{Injection time and dispensed volume}

Activation of each AAI was filmed using a Casio/Exilim high-speed camera (HS EX-FS10, Norderstedt, Germany). The maximum frame rate per second was 210 . The number of frames in which a fluid jet was visible determined the injection (ejection) time. The volume dispensed (into air) was calculated by subtracting the weight of the AAI after activation from its weight before activation. The weight difference in grams relates directly to the volume dispensed ( $1 \mathrm{~g}$ weight lost $=1 \mathrm{~mL}$ of fluid dispensed).

\section{Exposed needle length}

The length ( $\mathrm{mm}$ ) of the needle exposed from each AAI after activation was measured using a dial gauge attached to a specialist holding fixture (Figure 2). To simulate a true-tolife usage situation, the AAI was loaded with $20 \mathrm{~N}$ (ie, the force required for activation) applied using a Zwick testing machine.

\section{Performance of AAls in a gelatin model}

The second series of tests investigated the distribution of the injected volume over a period of 10 seconds and the depth of the injection into ballistic gelatin (a reliable soft tissue simulant used in wound ballistic experiments ${ }^{12}$ ).

\section{Distribution of solution into gelatin}

The distribution of the injected solution into ballistic gelatin (mixture ratio: 20vol\% gelatin/80vol\% water; see Schwirtz and Seeger ${ }^{11}$ for preparation instructions) was tested by analyzing a series of photographs taken during activation, using an

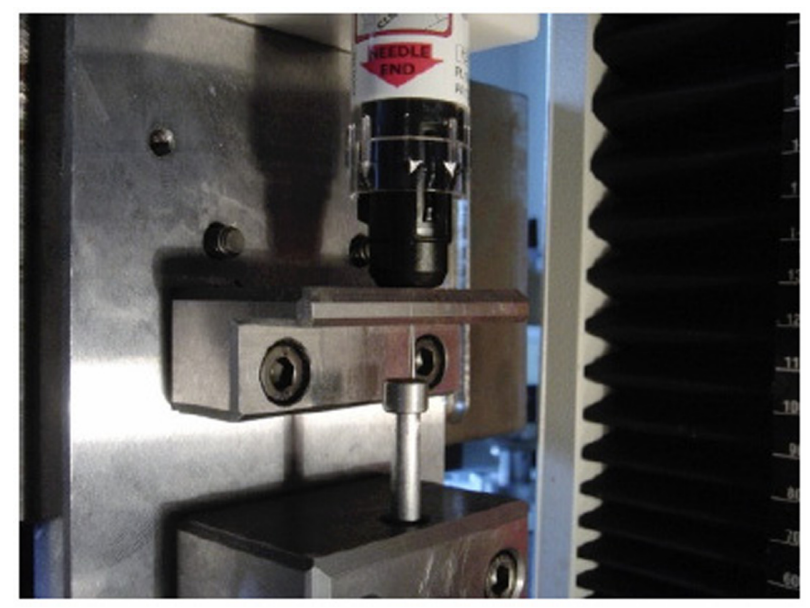

Figure 2 Apparatus for the measurement of exposed needle length.

Notes: The length of the needle exposed was measured using a dial gauge attached to a specialist holding fixture. Each adrenaline auto-injector was loaded with a force of $20 \mathrm{~N}$ (the force required for activation). 
application programmed in Matlab $^{\odot}$ (version R2008A, The Mathworks Inc, Natick, MA). To visualize the ejected solution, the clear adrenaline solution in each AAI device was replaced with black ink (Pelikan ${ }^{\odot} 4001$ Brilliant black). To replace the solution, the AAIs were disassembled, the original adrenaline solution was completely removed and replaced by ink injected into the device, and, afterwards, the injectors were reassembled (Figure 3 ). The procedure was essentially the same for all three devices.

The injection of ink from each device into the transparent block of ballistic gelatin was then filmed. For each frame of the film sequence, the colored area was calculated by Matlab digital image processing. The percentage of the colored area was calculated based on a $100 \%$ reference area for the distribution of a standard solution into gelatin and was defined as the average of the colored area delivered by the AAI after 10 seconds.

\section{Total injection depth in gelatin}

The total injection depth was defined as the maximum depth reached by the ink solution within 10 seconds after activation of the AAI into the gelatin block. The vertical distance from the surface of the gelatin to the lowest part of the ink area was measured by digital image processing and expressed in $\mathrm{mm}$.

\section{Statistics}

Under base conditions, 25 samples of each AAI were measured (Jext batch 000139318 [ $\mathrm{n}=25]$; EpiPen batches 0000129760-0000131211 [n=5], 0000132207-0000132894 $[\mathrm{n}=11], 0000130394-0000132454$ [ $\mathrm{n}=1], 0000132207-$ 0000132953 [n $=8$ ]; Anapen batches LJR14022012 $[\mathrm{n}=15]$, TLB10042013 [ $\mathrm{n}=8]$, LFW14022012 [n=1], MPD28032012 [n=1]). After being subjected to free-fall and static load conditions, 15 samples of each AAI were measured (free-fall: Jext batch 000139318 [n=15]; EpiPen batches 0000132207-0000132894 [ $\mathrm{n}=12$ ], 00001322070000132953 [n $=3$ ]; Anapen batches LJR14022012 $[\mathrm{n}=12]$, TLB10042013 [n=2], RRT14112012 [n=1]. Static load: Jext batch 000139318 [n $=15]$; EpiPen batches 0000132207-0000132894 [n=11], 00001322070000132953 [ $\mathrm{n}=4]$; Anapen batches LJR14022012 $[\mathrm{n}=8]$, TLB10042013 [ $\mathrm{n}=5]$, RRT14112012 [n = 1], LFW14022012 [n=1]). For the gelatin experiments, five samples of each AAI were used.

The statistical analyses were specified post hoc (after data collection). All statistical tests were two-sided at a $5 \%$ level of significance and all confidence intervals were two-sided $95 \%$ confidence intervals. Measures for centrality were reported as mean values and measures for variability were reported as a standard deviation. A Student's $t$-test with Satterthwaite's adjustment for the degree of freedom was used for hypothesis testing of equal means. No adjustment for multiple testing was performed.

In the box plots, the bottom and top edges of the box indicate the interquartile range (IQR, ie, the range of values between the 25 th and 75 th percentiles). The line inside the box indicates the median value and the diamond indicates the mean value. The whiskers that extend from each box indicate the range of values within a distance of 1.5 IQR from the median. Points indicated by circles are considered to be outliers. All values, including outliers, are included in the statistical calculations.
A

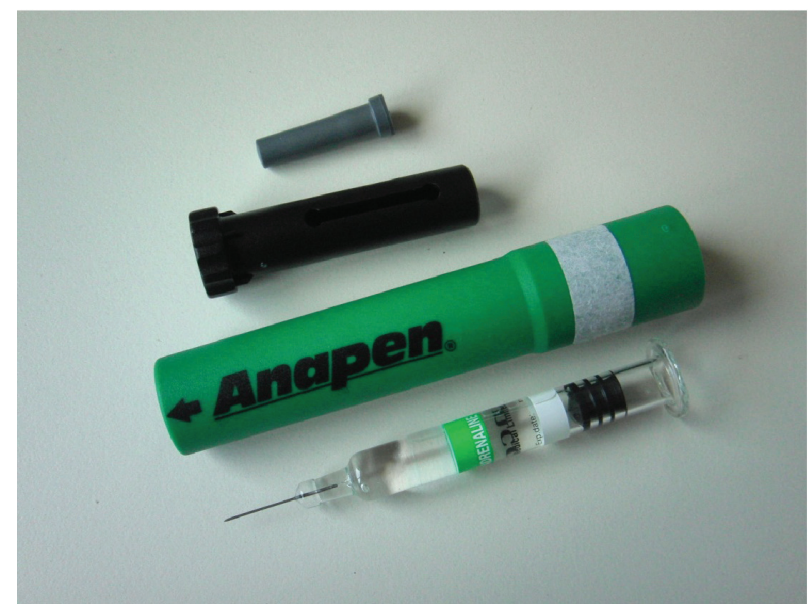

B

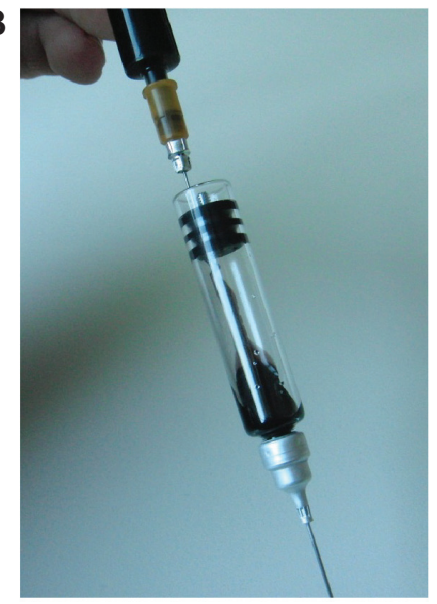

Figure 3 Preparation for gelatin distribution experiments. Prior to injection into gelatin, each device was disassembled (A) and the original adrenaline solution completely removed and replaced with ink (B). 


\section{Results}

\section{Robustness and functionality of AAls}

The force required to remove the safety cap from each device is shown in Figure 4. Significantly less force was required to remove the safety cap from Jext and EpiPen compared with Anapen under base conditions $(P<0.001$ for both comparisons). Following free-fall drops, the mean force needed to remove the safety cap from any of the AAIs was unchanged, whereas the application of a static load significantly increased the mean force required to remove the safety cap from Jext (difference from base value $7.7 \mathrm{~N}$; $P<0.001$ ) and from EpiPen (difference from base value $30.3 \mathrm{~N} ; P<0.001)$.

An additional analysis was carried out using the highest force required to remove the needle cap or the safety cap from Anapen. This analysis increased the mean force needed to remove the caps from Anapen at base conditions from 33.4 N (IQR 29.6, 37.2 N) to 35.3 N (IQR 31.9, 38.7 N; not statistically significant). Similar increases were seen when this analysis was performed for Anapens exposed to free-fall or static load conditions.

\section{Activation force}

The force required to activate the AAIs under the laboratory settings used (via exchangeable adapters that incorporated the different systems) was considered consistently low for all the AAIs (all observations were below 50 N, Figure 5). The free-fall conditions did not alter the force needed to activate any of the AAIs, whereas the static load significantly increased the force required to activate Anapen (adjusted mean difference from base $76.5 \mathrm{~N}, P=0.03$ ).

As shown in Figure 5, the static load resulted in outlier values for both EpiPen and Anapen, where the force needed to activate the AAIs increased to up to $450 \mathrm{~N}$. For one Anapen (6.7\%), the static load conditions increased the force needed to activate the device to $>500 \mathrm{~N}$ (equivalent to a $51 \mathrm{~kg}$ pressure), which was above the upper limit for the testing equipment (value not included in Figure 5). For one additional Anapen (6.7\%), the free-fall conditions resulted in a broken carpoule, making activation/injection impossible. The expiry date for these Anapens was February 14, 2012, which was approximately one month after the study was completed.

\section{Injection time and dispensed volume}

The mean values of the injection times (into air) and the derived volumes dispensed at base conditions for each of the AAIs are displayed in Table 1. The free-fall and static load tests did not alter to a significant degree the total volume dispensed by the AAIs or the injection time (data not shown).

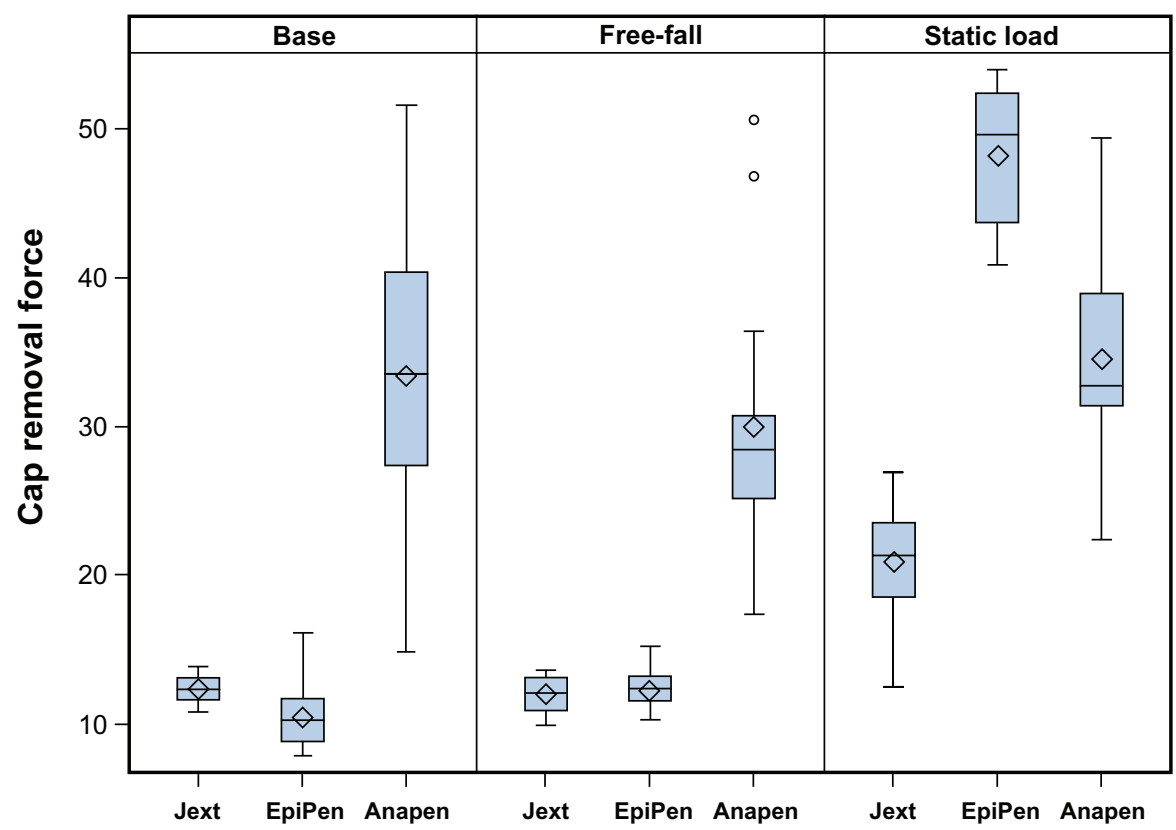

Figure 4 Box plots showing the force required $(\mathrm{N})$ to remove the safety cap from each adrenaline auto-injector at base conditions, after free-fall, and after static load preconditioning.

Notes: Median (line inside box), mean (diamonds), and outlying values (circles) demonstrate that the least amount of force was required to remove the safety cap from Jext and EpiPen under base conditions and after free-fall, which was significantly increased following static load $(P<0.00 \mathrm{I})$. 


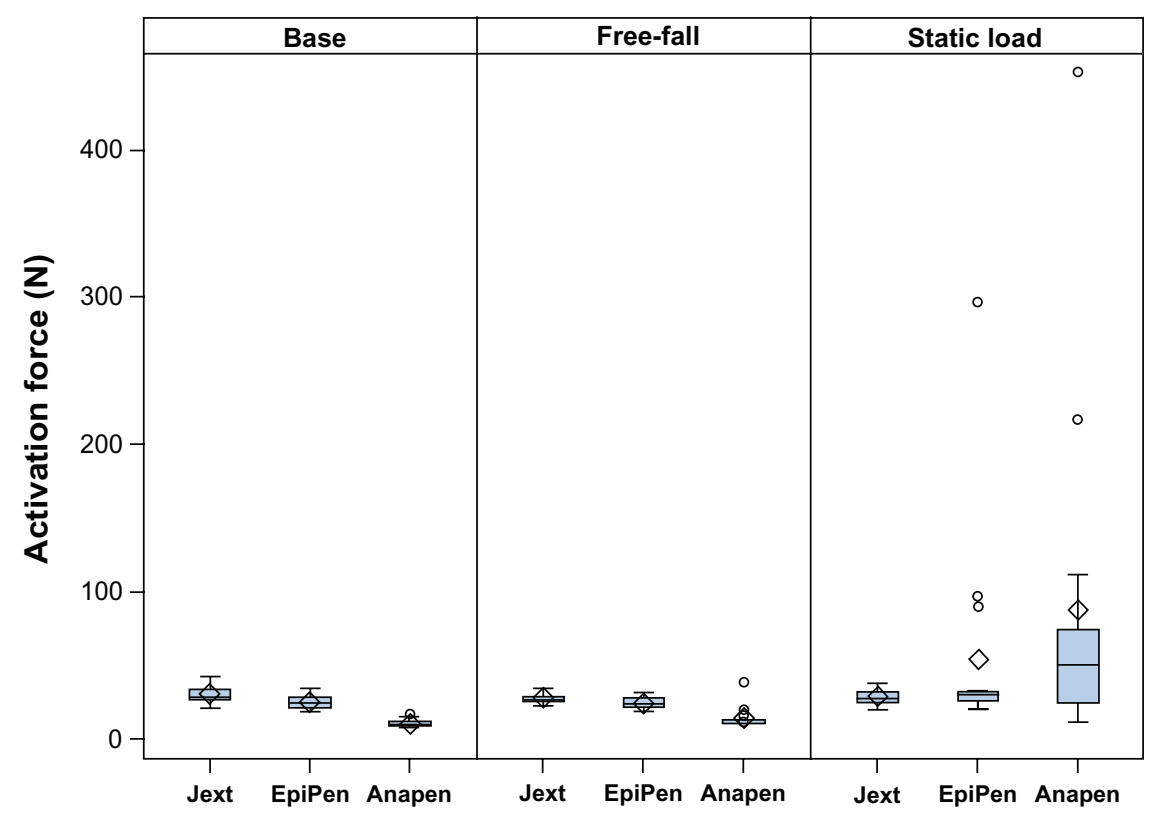

Figure 5 Box plots showing the force required $(\mathrm{N})$ to activate each adrenaline auto-injector at base conditions, after free-fall, and after static load preconditioning. Notes: Median (line inside box), mean (diamonds), and outlying values (circles) demonstrate that the static load significantly increased the force needed to activate EpiPen and Anapen. The force needed to activate one Anapen was above the upper limit for the testing equipment. The free-fall conditions resulted in a broken carpoule of another Anapen.

\section{Exposed needle length}

The mean exposed needle length was $15.36 \mathrm{~mm}$ (standard deviation [SD] 0.22) for Jext, $15.02 \mathrm{~mm}$ (SD 0.25) for EpiPen, and $7.49 \mathrm{~mm}$ (SD 0.73) for Anapen (Figure 6). The free-fall vertical test conditions caused bent needle tips on all EpiPens (Figure 7) and an accordingly reduced needle length (mean difference from base $-0.45 \mathrm{~mm} ; P<0.001$ ). The bent needle tips were not visible prior to activation, but closer examination revealed that the rubber seal of each EpiPen was pierced by the bent needle after the free-fall tests (Figure 7). Free-fall conditions did not affect the exposed needle length of Jext or Anapens. The static load did not

Table I Mean injection time into air (seconds) and mean derived volume dispensed $(\mathrm{mg})$ at base conditions for each AAI

\begin{tabular}{|c|c|c|c|}
\hline AAl type & Mean & SD & $95 \% \mathrm{Cl}$ \\
\hline \multicolumn{4}{|c|}{ Injection time (seconds) } \\
\hline Jext & 0.17 & 0.006 & {$[0.17-0.17]$} \\
\hline EpiPen & 0.19 & 0.017 & {$[0.18-0.19]$} \\
\hline Anapen & 1.16 & 0.089 & {$[1.13-1.20]$} \\
\hline \multicolumn{4}{|c|}{ Volume dispensed (mg) } \\
\hline Jext & 0.31 & 0.008 & {$[0.30-0.3 I]$} \\
\hline EpiPen & 0.30 & 0.016 & {$[0.29-0.30]$} \\
\hline Anapen & 0.29 & 0.023 & {$[0.28-0.29]$} \\
\hline
\end{tabular}

Notes: Jext displayed the shortest injection time and the greatest volume dispensed, followed by EpiPen and Anapen. 15 AAls of each type were tested.

Abbreviations: SD, standard deviation; $\mathrm{Cl}$, confidence intervals; AAl, adrenaline auto-injector. result in any significant change in the exposed needle length of any of the AAIs tested.

\section{Performance of AAls in a gelatin model}

The distribution of the injected solution into ballistic gelatin over 10 seconds for each type of AAI is shown in Figure 8. The estimated volume of the dispensed solution was based on the pixel area of the dispensed solution as a percentage of the reference area (average pixel area after 10 seconds; Figure 9). While almost $50 \%$ of the reference area was reached by Jext and EpiPen within 0.5 seconds, the estimated volume injected with Anapen only reached $5 \%$ of the

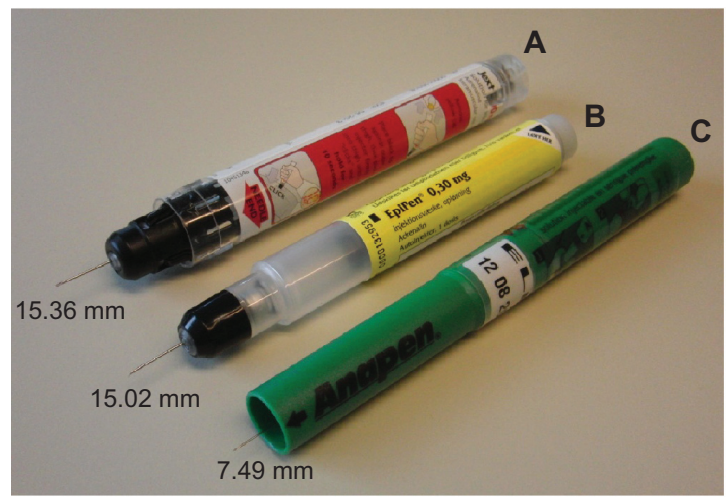

Figure 6 Exposed needle lengths $(\mathrm{mm})$ of Jext $(\mathbf{A})$, note that the black needle protection tip is manually retracted to show the needle, EpiPen (B), and Anapen (C).

Note: Jext had the longest exposed needle length, followed by EpiPen and Anapen. 

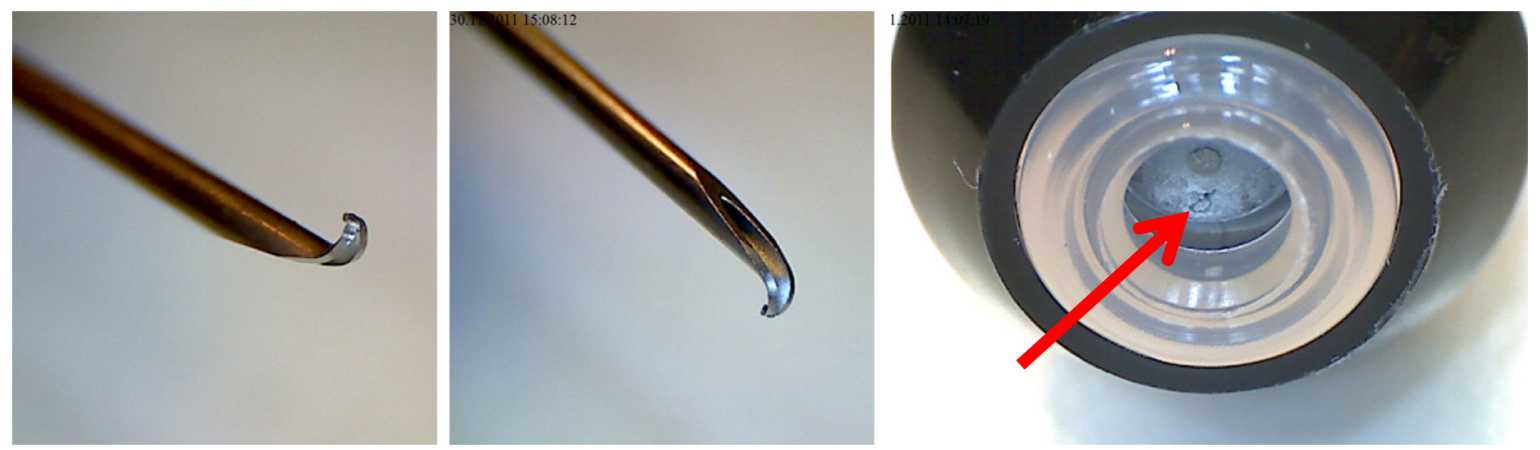

Figure 7 Images of an EpiPen following a $1.5 \mathrm{~m}$ free-fall test.

Note: Bent needle tips, accordingly reduced needle lengths $(P<0.00 \mathrm{I})$, and pierced rubber sealings (red arrow) were evident on all EpiPens.

reference area within 0.5 seconds and less than $60 \%$ of the reference area within 10 seconds.

\section{Total injection depth in gelatin}

The mean maximum injection depth in gelatin within 10 seconds was $28.87 \mathrm{~mm}$ (SD 0.73) for Jext, $29.68 \mathrm{~mm}$ (SD 2.08) for EpiPen, and $18.74 \mathrm{~mm}$ (SD 1.25) for Anapen (Figure 10). The injection depth was significantly shallower for Anapen than for EpiPen and Jext ( $P>0.001$ for both comparisons). There was no significant difference in injection depth between EpiPen and Jext $(P=0.45)$.

\section{Discussion}

The optimal management of anaphylaxis requires immediate intramuscular injection of adrenaline using an AAI. In order to ensure optimal functionality, the characteristics of the "ideal" AAI have been postulated. ${ }^{9}$ These include delivering the correct dose of adrenaline to the correct tissue compartment within the correct time frame; as well as being easy, convenient, robust, safe and reliable enough to withstand real-life storage and use. ${ }^{9}$

In this study, the robustness and functionality of three different AAIs available in Europe were compared: two cartridge-based AAIs (Jext and EpiPen) and one syringebased AAI (Anapen). The injection performance of the AAIs was also compared using ballistic gelatin (a reliable soft tissue simulant).

Data from this study showed significant advantages of the cartridge-based AAIs (Jext and EpiPen) compared with the syringe-based AAI (Anapen) in terms of both robustness and the appropriate delivery of solution into the ballistic gelatin. It is noteworthy that Jext was the only cartridge-based AAI that was $100 \%$ functional after robustness tests. However, only one batch of Jext AAIs was tested in the study, which was the first commercial batch available, compared with five different batches of Anapen and four different batches of EpiPen. This
0.5 seconds

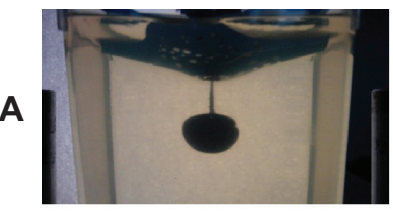

B

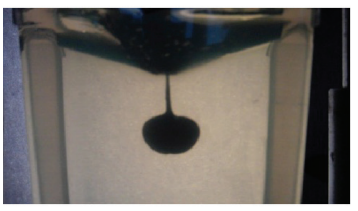

C

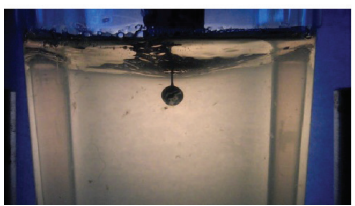

2.5 seconds
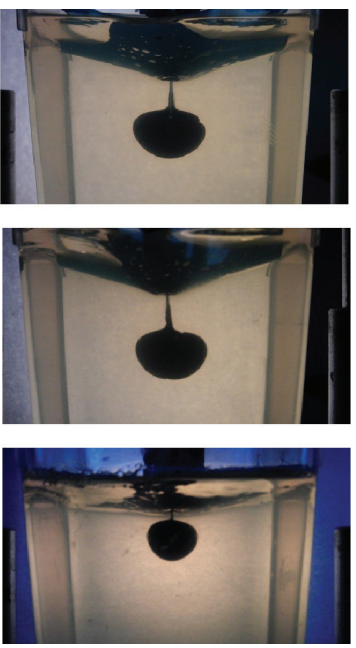

5 seconds
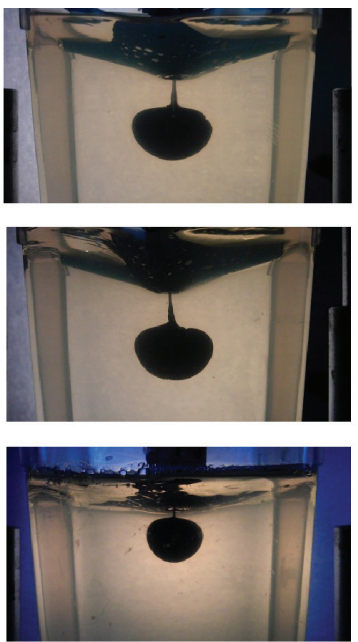

10 seconds
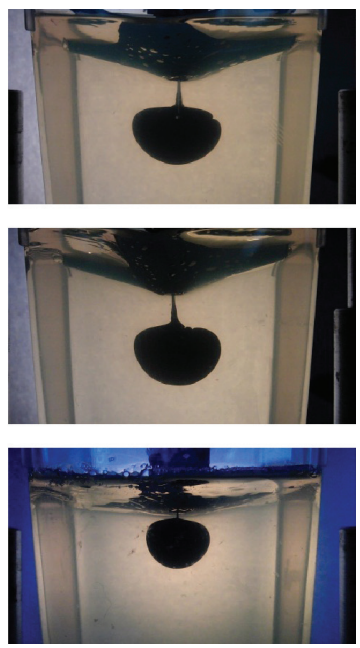

Figure 8 Photographs of solution dispensed into gelatin using Jext (A), EpiPen (B), and Anapen (C) at 0.5, I.0, 5.0, and I0.0 seconds after activation. 


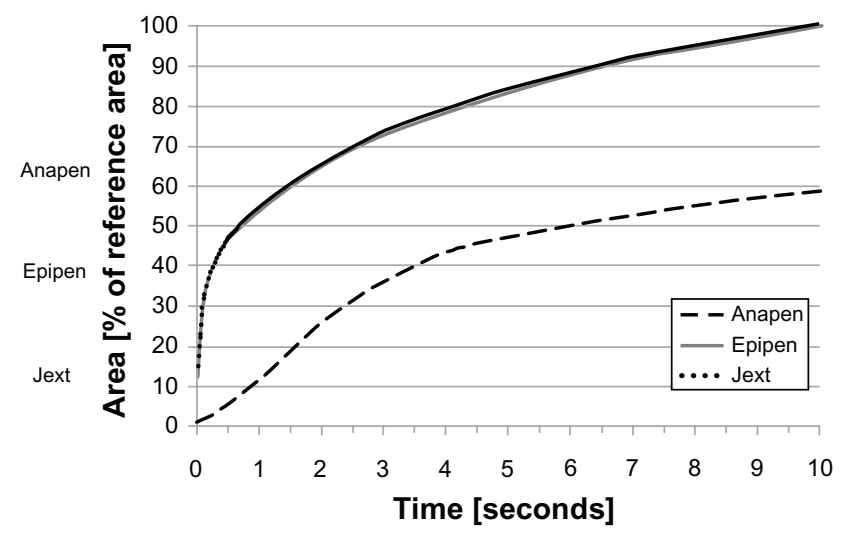

Figure 9 Graph showing the estimated area (volume) of the dispensed solution from the AAls into gelatine over time (seconds), based on pixel area (digital image processing) from a series of photographs taken during activation (see Material and methods for a full explanation).

Note: Almost $50 \%$ of the reference area was reached by Jext and EpiPen within 0.5 seconds, compared with $5 \%$ for Anapen.

may explain the noticeably lower variation in functionality that was observed with Jext. It is also pertinent to highlight that all models used in this study are simplified simulations of real-world conditions, which may be much more varied. No intrabatch or interbatch variability was observed during the study.

An essential feature of an AAI is its ability to operate reliably after being carried and subjected to the stresses of everyday life. ${ }^{9}$ In the current study, the application of a $40 \mathrm{~kg}$ weight significantly increased the force required to activate Anapen and EpiPen. It is noteworthy that this static load increased the force required to activate one Anapen (6.7\%) to beyond 500 N. Such an activation force would render the device extremely difficult to use, especially for pediatric patients. Additionally, two in-date Anapens (13.3\%) and one EpiPen (6.7\%) required a force of more than $200 \mathrm{~N} / 20 \mathrm{~kg}$ to be activated after being subjected to the static load conditions. The maximum downward thumb pushing force has been found to be around $200 \mathrm{~N}$ (dependent on age and gender). ${ }^{13}$ Because an AAI should be carried at all times and will, over its lifetime, be subjected to high levels of stress that may mimic the static load conditions used in the study, such an outcome could occur in a real-world situation, where the inability to activate an AAI could have far-reaching or fatal consequences. ${ }^{7}$

These findings are in general agreement with those obtained in a pilot study comparing the mechanical and injection performance characteristics of cartridge-based and syringe-based AAIs for pediatric use. ${ }^{11}$ There is one discrepancy between our data and that from the pilot study. Although the device mechanisms of the senior and junior AAIs are identical, in the pilot study, when subjected to static load conditions similar to those used in the current study, $100 \%$ of EpiPen Junior devices and $86.7 \%$ of Anapen Junior devices were damaged to a degree that made them impossible to activate. ${ }^{11}$ The variation in AAI robustness between the pilot study and the current analysis is likely a result of variations between individual device batches.

Both studies also shared identical test methodology. However, the manufacturer's expiry date on the EpiPen batches used in the pilot study was October 2008, making these AAIs beyond their expiry date when the study was conducted. In contrast, it was ensured that the devices included in the current study were within their expiry date. Upon further inspection, the EpiPen device housing appeared more brittle than standard on the out-of-date AAIs and, as a result, more susceptible to failure following free-fall drop and static load tests. These data reinforce the necessity of replacing AAIs within the manufacturer's expiry date (ie, 24 months from the date of manufacture for Jext, 18 months for EpiPen, and 21-24 months for Anapen).
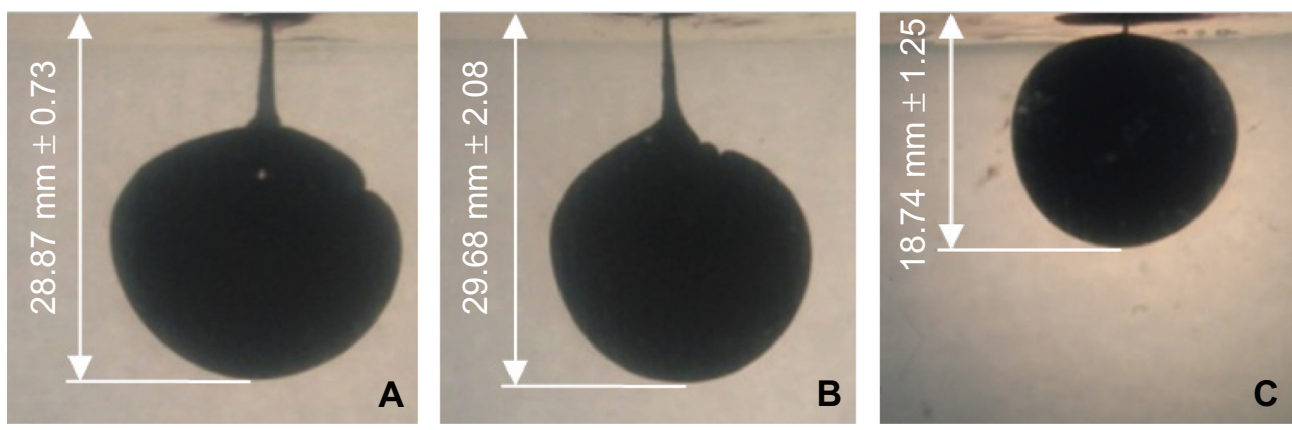

Figure 10 Photographs showing the total injection depth into gelatin 10 seconds after activation of Jext (A), EpiPen (B), and Anapen (C), measured as the vertical distance from the surface of the gelatin to the lowest part of the ink area using digital image processing.

Note: The injection depth was significantly shallower for Anapen than for EpiPen and Jext $(P<0.00 \mathrm{I}$ for both comparisons). 
A second difference between the EpiPens used in the pilot study and the current study is the materials used in their manufacture. In 2010, the manufacturer of EpiPen reported a change to the plastic materials used in the device housing in order to improve strength and consistency. Hence, devices manufactured prior to 2010 are likely to be more susceptible to critical damage.

The free-fall conditions applied in this study (from a height of $1.5 \mathrm{~m}$ ) led to a broken carpoule in one Anapen (6.7\%), making the AAI dysfunctional. This was the only immediate clinically significant consequence of the applied free-fall conditions. The free-fall conditions also led to bent needle tips on all EpiPens. Although this most likely would not affect the possibility of injection and only slightly reduced the potential depth of the injection (exposed needle length decreased by $0.45 \mathrm{~mm}$ ), it may be associated with more pain when removing the needle from the thigh after injection. The needle also pierced the rubber seal of the AAI, which could potentially lead to contamination of the needle tip. Although infection after intramuscular injection is rare, injection using a contaminated needle may have fatal

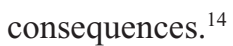

A substantial barrier to the correct use of AAIs is the patient's ability to hold the device in place during the 10 seconds that is normally recommended for adrenaline injection. ${ }^{15}$ In this study, we found that the complete injection time into air was less than 1.2 seconds for all AAIs. However, injection into gelatin was substantially slower, in particular for Anapen where the volume injected after 10 seconds was less than $60 \%$ of the reference area. This finding raises concerns about the ability of Anapen to deliver the required dose of adrenaline. For Jext and EpiPen, almost $50 \%$ of the volume was injected within 0.5 seconds, compared with $5 \%$ for Anapen.

Injection depth is another important factor in the delivery of the correct dose of adrenaline to the correct tissue compartment. The effective penetration depth of an AAI is a function of the syringe force, which is influenced by the power of the spring contained within the device, needle length, needle bore diameter, and tissue characteristics. It has been suggested that the glass syringe contained within syringe-based AAIs limits the force that can be applied to the spring. ${ }^{16}$ In agreement, our study showed that when measuring both the exposed needle length and maximum injection depth into gelatin, EpiPen and Jext were superior to Anapen.

The distance from skin to muscle is a key tissue characteristic that influences AAI penetration. ${ }^{16}$ The mean skin-to-muscle distance has been estimated at $14.8 \mathrm{~mm}$ for women $(\mathrm{n}=50)$. Thus, a needle length shorter than this value may not be able to deliver adrenaline to the intramuscular tissue. ${ }^{17}$ In the current study, the maximal injection depth in gelatin after 10 seconds was above $15 \mathrm{~mm}$ for all AAIs, although this may be biased by the homogeneity of ballistic gelatin and the inherent limitations in mimicking human thigh tissue.

To limit this bias, we have developed a model in which the contents of AAIs are replaced with a contrast agent, the AAI is activated into fresh pork shoulder, and computer tomography scanning is used to examine the injection pathway. This model has been used to assess the injection depth of syringe-based (Anapen $300[\mathrm{n}=4]$ and 500 $[\mathrm{n}=3]$ ) versus cartridge-based (EpiPen $[\mathrm{n}=3]$ and Jext $[\mathrm{n}=4])$ AAIs. The maximum injection depth ranged from $8.9 \mathrm{~mm}$ to $9.9 \mathrm{~mm}$ for Anapen compared with $14.5 \mathrm{~mm}$ to $15.2 \mathrm{~mm}$ for Jext and EpiPen. Three of seven Anapens did not penetrate the muscle layer of the pork shoulder, whereas all cartridge-based AAIs did penetrate the muscle. Finally, the maximum injection depth of Anapen failed to reach the muscle each time the skin-to-muscle distance was greater than the exposed needle length of the AAI. The maximum injection depth reached by Jext and EpiPen always reached muscle, even when the skin-to-muscle distance was greater than the exposed needle length. Hence, using this model, Anapen appears to be unable to inject deeper than its exposed needle length. ${ }^{18}$

Previous evidence supports other advantages of cartridgebased AAIs over the syringe-based system, such as a simpler operation sequence. Indeed, Anapen requires an extra operational step where the needle cap, including a needle protective shield, must be detached before removal of the safety cap. An extra operational step may increase the risk of misuse because patients have to memorize and undertake the correct operational sequence at a time of high stress and urgency. ${ }^{9}$ Despite this, in a study of EpiPen and Anapen that included 100 mothers, a significantly higher proportion correctly performed Anapen-specific injection procedures $(P \leq 0.0001) .{ }^{19}$ Thus, patient preference and other factors clearly influence AAI performance.

Additional variability in the performance of AAIs may be introduced by the injection technique used by patients. For example, there are two distinct techniques generally taught by prescribers and used by patients to activate cartridgebased AAIs, ie, the "swing and jab" technique, as described in the current EpiPen summary of product characteristics, 
and the "place and press" technique, as described in the Jext summary of product characteristics. In a separate study, we compared the injection depth, injection volume, and exposed needle length of Jext following activation by each of these two techniques. This study demonstrated that Jext can be activated using either the place and press method or the swing and jab method; with comparable exposed needle length, injection depth, and dose volume achieved irrespective of injection technique. ${ }^{20}$ Therefore, patients switched from EpiPen to Jext do not need to be retrained with regards to the AAI activation sequence or steps. However, regular AAI training should be part of an individual anaphylaxis management plan. ${ }^{20}$

In the current study, Jext performed better than EpiPen following robustness tests. At the time of writing, new versions of Anapen and EpiPen have been approved in some European countries. Based on the approved summaries of product characteristics, these new devices appear to have similar physical properties to those assessed in this study. Therefore, we do not expect any major differences versus the original devices regarding the features investigated in this study. This hypothesis will be tested once these new devices are commercially available. Additional studies may also be required to assess the ease-of-use and convenience of individual AAIs, because these characteristics have a significant influence on the functionality of the devices.

\section{Conclusion}

It is essential that AAIs are effective and reliable throughout their shelf-life to ensure they are fit for their life-saving purpose. A comparison of the robustness, functionality, and performance (in a gelatin tissue model) of three AAIs showed that cartridge-based AAIs (Jext and EpiPen) appeared to be significantly more robust and more capable of quickly and consistently delivering the correct dose of adrenaline to the correct tissue compartment than syringe-based AAIs (Anapen). Overall, Jext performed better than EpiPen and Anapen following mechanical stress tests designed to mimic real-world conditions. Because this study focused on assessing if AAIs function correctly and reliably, further research is recommended to compare AAIs in terms of ease-of-use and convenience.

\section{Acknowledgments}

The authors would like to thank Markus Csenar and Simon Hendry for input regarding the test settings and the study design, and D Rehm and B Riis of ALK-Abelló for providing statistical and medical writing assistance.

\section{Disclosure}

The study was sponsored by ALK AG. AS and HS are employees of Pharma Consult, a company which can collect payments from Jext performance. AS and HS were responsible for the design and conduct of the study. AS helped to design Jext.

\section{References}

1. Kim H, Fischer D. Anaphylaxis. Allergy Asthma Clin Immunol. 2011; 7 Suppl 1:S6.

2. McLean-Tooke AP, Bethune CA, Fay AC, Spickett GP. Adrenaline in the treatment of anaphylaxis: what is the evidence? BMJ. 2003; 327:1332-1335

3. Lieberman P, Nicklas RA, Oppenheimer J, et al. The diagnosis and management of anaphylaxis practice parameter: 2010 update. JAllergy Clin Immunol. 2010;126:477-480.

4. Lieberman P, Camargo CA Jr, Bohlke K, et al. Epidemiology of anaphylaxis: findings of the American College of Allergy, Asthma and Immunology Epidemiology of Anaphylaxis Working Group. Ann Allergy Asthma Immunol. 2006;97:596-602.

5. Gupta R, Sheikh A, Strachan DP, Anderson HR. Time trends in allergic disorders in the UK. Thorax. 2007;62:91-96.

6. Simons FE, Ardusso LRF, Biló MB, et al. World Allergy Organization guidelines for the assessment and management of anaphylaxis. World Allergy Organiz J. 2011;127:587-593. e1-e22.

7. Simons FE, Lieberman PL, Read EJ Jr, Edwards ES. Hazards of unintentional injection of epinephrine from autoinjectors: a systematic review. Ann Allergy Asthma Immunol. 2009;102:282-287.

8. Simons FE. Lack of worldwide availability of epinephrine autoinjectors for outpatients at risk of anaphylaxis. Ann Allergy Asthma Immunol. 2005;94:534-538.

9. Frew AJ. What are the 'ideal' features of an adrenaline (epinephrine) auto-injector in the treatment of anaphylaxis? Allergy. 2011;66: $15-24$.

10. ALK-Abello A/S press release. ALK launches Jext ${ }^{\circledR}$, a new adrenaline auto-injector. 2011. Available at: http://www.life-saver.org/wordpress/ wp-content/uploads/2011/09/Medical-Jext-PR-FINAL.pdf. Accessed June 27, 2012.

11. Schwirtz A, Seeger H. Are adrenaline autoinjectors fit for purpose? A pilot study of the mechanical and injection performance characteristics of a cartridge-versus a syringe-based autoinjector. $J$ Asthma Allergy. 2010;3:159-167.

12. Jussila J. Preparing ballistic gelatine - review and proposal for a standard method. Forensic Sci Int. 2004;141:91-98.

13. Peebles L, Norris B. Filling 'gaps' in strength data for design. Appl Ergon. 2003;34:73-88.

14. Stuart Hannah RC, Heddle R, Smith W. Fatal gas gangrene related to self-injection treatment of anaphylaxis. Ann Allergy Asthma Immunol. 2011;106:538.

15. Mehr S, Robinson M, Tang M. Doctor- - how do I use my EpiPen? Pediatr Allergy Immunol. 2007;18:448-452.

16. Ram FSF. Self-injectable adrenaline for anaphylactic emergencies: effectiveness of Epipen and Anapen - are they fit for purpose? J Aller Ther. 2012;3:1-4.

17. Song TT, Nelson MR, Chang JH, Engler RJ, Chowdhury BA. Adequacy of the epinephrine autoinjector needle length in delivering epinephrine to the intramuscular tissues. Ann Allergy Asthma Immunol. 2005;94:539-542. 
18. Schwirtz A. Anaphylaxis treatment: comparison of the injection depth of 3 adrenaline auto-injectors in a pork shoulder model [abstract]. Presented at the European Academy of Allergy and Clinical Immunology 31st Annual Meeting, Geneva, Switzerland; June 16-20, 2012:1683.

19. Brown JDM, Spear E, Alfaham M, Tuthill D. A randomised maternal evaluation of epinephrine auto-injection devices. Arch Dis Child. 2011;96:A49.
20. Seeger H. Comparison of 'place and press' and 'swing and jab' activation of a novel cartridge-based adrenaline auto-injector [abstract]. Presented at the European Academy of Allergy and Clinical Immunology 31st Annual Meeting, Geneva, Switzerland; June 16-20, 2012:1684.

\section{Publish your work in this journal}

The Journal of Asthma and Allergy is an international, peer-reviewed open-access journal publishing original research, reports, editorials and commentaries on the following topics: Asthma; Pulmonary physiology; Asthma related clinical health; Clinical immunology and the immunological basis of disease; Pharmacological interventions and

\section{Dovepress}

new therapies. Issues of patient safety and quality of care will also be considered. The manuscript management system is completely online and includes a very quick and fair peer-review system, which is all easy to use. Visit http://www.dovepress.com/testimonials.php to read real quotes from published authors.

Submit your manuscript here: http://www.dovepress.com/journal-of-asthma-and-allergy-journal 